\title{
Reliability and response assessment of brain responses in fNIRS
}

\author{
XiAOWel JiAng, ${ }^{3}$ Y ANAN Chen, Ph.D, $1,2,{ }^{*}$ Chenghao Zhou, ${ }^{1}$ NA AO 1 \\ 1 Institute of Psychology and Behavior, Henan University, Kaifeng, China \\ 2 Institute of Cognition, Brain and Health, Henan University, Kaifeng, China \\ 3 Department of Bioengineering, University of Pennsylvania, Philadelphia, PA, United States Address \\ *henyn@henu.edu.cn
}

Highlight: Based on the advantages of fNIRS, this paper designed a new method, named the Area-Under-Curvebased Indicator System (AUCIS), to identify different brain responses, thus explaining the brain activity by physiological mechanism. At the same time, it is possible to verify the robustness of the effect.

\begin{abstract}
:
Background: Functional near-infrared spectroscopy (fNIRS) is a new noninvasive neuroimaging technology that detects both oxyhemoglobin hemodynamics $(\mathrm{HbO})$ and deoxy-hemoglobin hemodynamics $(\mathrm{HbR})$, but there is no assessment approach that emphasizes the merits of fNIRS.

New method: Based on fNIRS, we established an indicator system named the Area-Under-Curve-based Indicator System (AUCIS) to estimate the effect reliability of brain responses. Evaluating the positive and negative responses for $\mathrm{HbO}$ and $\mathrm{HbR}$ can better explain, to some extent, the comprehensive physiological mechanism of oxygen delivery to and extraction in the brain. Moreover, we also established a reliability coefficient, named AUC' $\alpha$, to assess the robustness of within-subject condition effects.

Results and Comparison: To validate the AUCIS, we used a simulation-based HRF signal and an open database and compared the performance with other general indicators. The AUCIS showed a greater relative sensitivity and robustness, which can be explained in terms of oxygen delivery and extraction based on the negative and positive responses of $\mathrm{HbO}$ and $\mathrm{HbR}$.
\end{abstract}

Keyword: fNIRS, brain response, reliability coefficient, AUCIS

Funding: This research was supported by Institute of Psychology, CAS (No. GJ202007). 


\section{Introduction}

Functional near-infrared spectroscopy (fNIRS) is a new noninvasive neuroimaging technology(Calderon-Arnulphi et al., 2009). It was first used in clinical research to transilluminate the newborn head(Brazy et al., 1985). Hemoglobin molecules absorb near-infrared (NIR) spectral region light with different characteristic absorption spectra for oxyhemoglobin hemodynamics $(\mathrm{HbO})$ and deoxy-hemoglobin hemodynamics $(\mathrm{HbR})$ based on molecular chemical bonds(Cope et al., 1988). These variations in absorption can reveal the depth of recorded neural activation and localized cortical activity in real time(Montcel et al., 2006). Furthermore, the oxyhemoglobin dissociation curve shows the relationship of $\mathrm{HbO} 2$ and $\mathrm{Hb}$ and their conversion(Kaufman et al., 2021).

$$
\mathrm{Hb}+\mathrm{O}_{2} \leftrightarrow \mathrm{HbO}_{2}
$$

Consequently, based on the Beer-Lambert law(Pellicer \& Bravo, 2011) or the fixed-value modified Beer-Lambert law (MBBL)(Asgher et al., 2019), fNIRS can be used to reveal HbO and HbR responses(Calderon-Arnulphi et al., 2009; Cope et al., 1988; De Blasi et al., 1993; Hoshi, 2011; Pellicer \& Bravo, 2011), and they can often be observed as temporally coupled signals(N. D. Tam \& Zouridakis, 2013, 2014; N. Tam \& Zouridakis, 2013). The HbO signal represents oxygen delivery to tissues, and HbR represents oxygen extraction from the tissue(N. Tam et al., $2017 ; \mathrm{N}$. D. Tam \& Zouridakis, 2014). Neurovascular coupling correlates with neuronal activation and vascular responses(Toronov et al., 2001), and $\mathrm{HbO}$ and $\mathrm{HbR}$ has been experimentally shown to share this coupling(N. D. Tam \& Zouridakis, 2014). Therefore, fNIRS hemodynamic signals can quantify the temporal profiles of the neurovascular response from a cognition task in real time(N. D. Tam \& Zouridakis, 2014). Moreover, providing a smoother hemodynamic response function (HRF) curve during the task becomes available because of the better temporal resolution (msec) of fNIRS(Calderon-Arnulphi et al., 2009; Hoshi, 2011; Pellicer \& Bravo, 2011), especially compared with functional magnetic resonance imaging (fMRI).

fMRI usually acquires low-resolution temporal signals (1 second) to scan the whole brain. fMRI primarily identifies HbR levels, as blood oxygen level-dependent (BOLD) activity, rather than HbO levels because HbR molecules are more paramagnetic(Poldrack et al., 2011). The increased signal observed when a stimulus is applied implies a relative decrease in the concentration of paramagnetic deoxy-hemoglobin. The oxygen extraction fraction (OEF) and the cerebral metabolic rate of oxygen are key to understanding brain function(Fan et al., 2020), and OEF is much lower than the blood flow increase following stimulation found in an early positron emission tomography (PET) study(Baron \& Jones, 2012), which showed the influence of hemodynamic measurements for clinical application(Okazawa \& Kudo, 2009). Theoretically, brain hemodynamics can be quantified for measuring OEF(Yablonskiy et al., 2013). Therefore, BOLD activity can be detected more sensitively, reflecting pooled local field potential activity(Logothetis et al., 2001).

Although real-time cognitive tasks show robustness between subjects, such as the real-life situation(Mayseless et al., 2019), through virtual reality technology(Wang et al., 2019) and laboratory experiments(Curtin et al., 2019; Ochi et al., 2018), and there are some assessments to evaluate the channel quality, such as using the scalp coupling index (sci) to identify optical channels showing a strong cardiac signal(Pollonini et al., 2014), assessing optical intensity(Rosa et al., 2019; Rovetti et al., 2019; Yuan et al., 2020), determining signal-to-noise ratio (<2) (Rosa et al., 2019) as a bad skin-optode coupling channel to be discarded, or setting the $\Delta$ concentration threshold (recommended as $80 \mu \mathrm{mol} / \mathrm{L}$ by $\mathrm{MNE}($ Gramfort et al., 2014)), the robustness of effects across trials under the same conditions continues to be ignored. Moreover, there are fewer time-field indicators designed that are especially designed to emphasize fNIRS advantages. Generally, the first-choice measurements are to average the whole signal(Grossmann et al., 2018; Rovetti et al., 2019; Tse et al., 2018) and the maximum and minimum amplitudes as ported from electroencephalogram (EEG), and construct general linear models (GLMs)(Rosa et al., 2019; Sagiv et al., 2019; Wang et al., 2019; Ye et al., 2009) as ported from fMRI. Although these three indicators are accessible to calculate, there are some disadvantages. GLM only presents the total $\beta$ of one condition. Minimum amplitude can represent the negative response directly but ignores the positive response. In addition, the maximum amplitude can directly represent a positive response but ignores the negative response. To make matters worse, averaged amplitudes will eliminate both as it only shows a positive response minus a negative response.

Consequently, to better incorporate the positive and negative responses for $\mathrm{HbO}$ and $\mathrm{HbR}$, we calculated the area under the curve (AUC) by definite integral to estimate the positive and negative responses. We independently integrated the positive signal time fields for the whole task, which is in reference to the positive response and named the positive activation degree (PAD); similarly with the negative response, the negative fields were integrated and named the negative activation degree (NAD). The total response is the summation of PAD and NAD, named the total activation degree (TAD). These indicators consider dynamic changes, distinguish different responses, and present a 
relatively comprehensive performance of brain activity during task performance. Then, to estimate the state of the signals, the AUC ratios are calculated from PAD divided by TAD and NAD divided by TAD, which are named the positive activation ratio (PAR) and negative activation ratio (NAR), respectively. These ratios directly represent the possibility of the signal state, either a positive state or negative state, by setting a threshold such as 0.5 , which would indicate whether the positive response was larger than the negative response, or vice versa. To estimate the robustness of the effect across trials within the same condition, we use a proportion of positive and negative states to all trials to represent the condition channel robustness, and these are named the repeated stability of positive activation ratio (RSPAR) and repeated stability of negative activation ratio (RSNAR). Based on an assumption that one subject has high reliability in a particular condition if any channel has high robustness, the reliability coefficient of effect (AUC' $\alpha$ ) was defined as the maximum proportion of any state in any channel. The relationships within the AUCIS is shown in Fig 1(a).

(a)

(b)

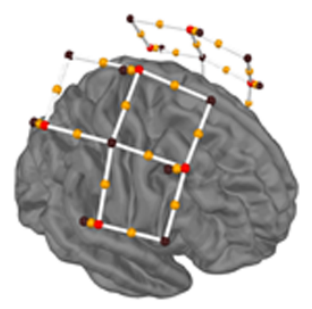

(c)

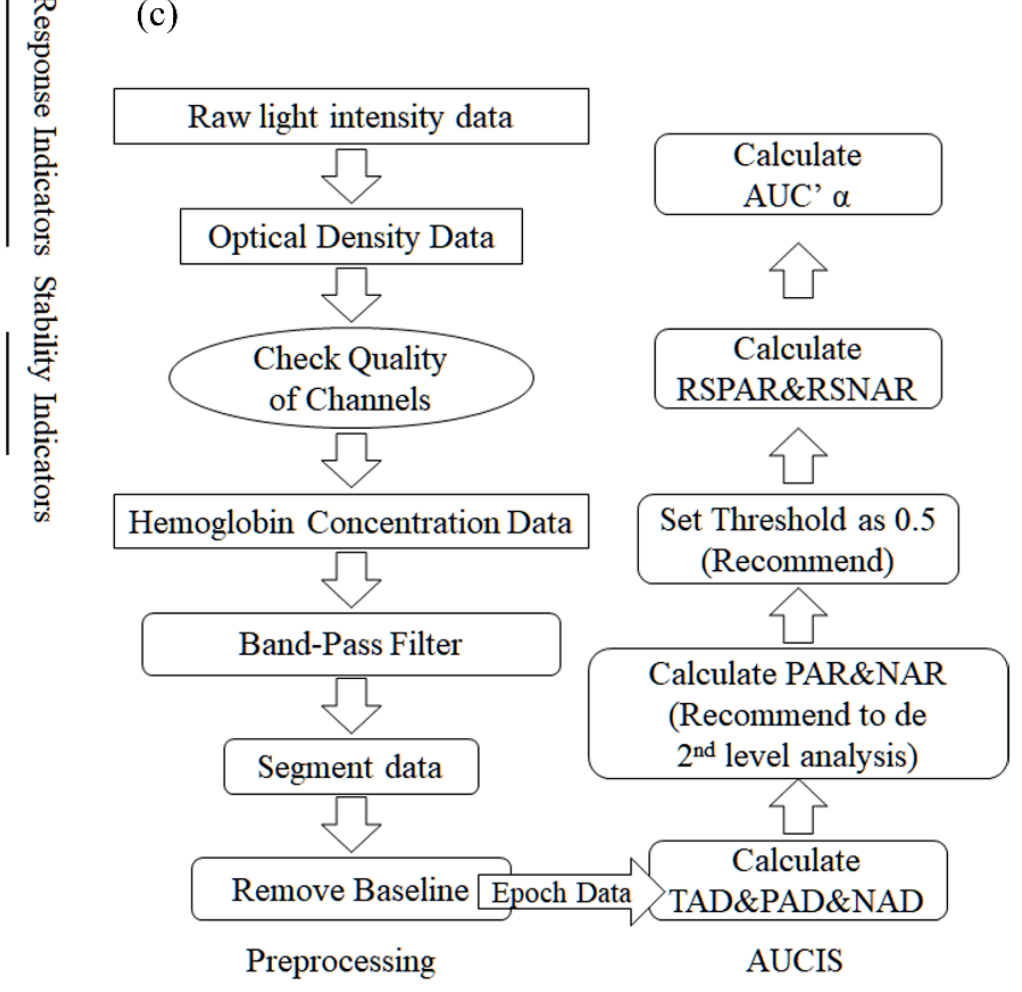

Fig 1 (a). The generating chain and structure of AUCIS. (b). The demo of source-detector pairs. The mid orange dots on the lines between the optodes show channels, red and black dots are referred to as sources and detectors. (c). The flow chart of preprocessing and $1^{\text {st }}$-level statistical analysis.

In summary, to better describe brain responses, we defined an Area-Under-Curve-based Indicator System (AUCIS) containing three parts: 5 response indicators including total activation degree (TAD), positive activation degree (PAD), negative activation degree (NAD), positive activation ratio (PAR), positive activation ratio (PAR), and negative activation ratio (NAR); 2 stability indicators including repeated stability of positive activation ratio (RSPAR) and repeated stability of negative activation ratio (RSNAR); and a reliability coefficient of effect (AUC' $\alpha$ ). We also used a simulation-based HRF signal to test how different parameters change the AUCIS. Then, we used the real signals from a finger-tapping task to estimate and validate the AUCIS and compare it with the general indicators, including the maximum, minimum and averaged amplitudes. 


\section{Definition}

\subsection{Response Indicators}

Positive Activation Degree (PAD) is an indicator referred to as the positive brain response. It ignores the negative response while focuses on the positive activity. The range is from 0 to infinite.

$$
\operatorname{PAD}=\int_{0}^{t m a x} \operatorname{Relu}(s(t)) d t
$$

Negative Activation Degree (NAD) is an indicator referred to as the negative brain response. It ignores the positive response while focuses on the negative activity. The range is from 0 to infinite.

$$
\mathrm{NAD}=\int_{0}^{t \max } \operatorname{Relu}(-s(t)) d t
$$

Total Activation Degree (TAD) is an indicator referred to as the total brain response. It contains both positive and negative responses, adding them to assume the total brain activation by task. The range is from 0 to infinite.

$$
\mathrm{TAD}=\int_{0}^{t m a x}|s(t)| d t=\mathrm{PAD}+\mathrm{NAD}
$$

Where tmax is the maximum time of the task, $s(t)$ is the BOLD signal, and the Relu algorithm can transform the negative number to zero and keep the positive number unchangeable. Fig 2(a) shows the PAD and NAD.

Positive Activation Ratio (PAR) represents the proportion of positive response in total response. This signal is defined as a positive response signal if PAR is over a threshold like 0.5 or 0.7 .

$$
\mathrm{PAR}=\frac{\mathrm{PAD}}{T A D}
$$

Negative Activation Ratio (NAR) represents the proportion of negative response in total response. This signal is defined as a negative response signal if NAR is over a threshold like 0.5 or 0.7 .

$$
\mathrm{NAR}=\frac{\mathrm{NAD}}{T A D}
$$

\subsection{Stability Indicators}

Repeated Stability of Positive Activation Ratio (RSPAR) represents the proportion of positive responses during the experiment.

$$
\text { RSPAR }=\frac{N_{\text {positive }}}{N}
$$

Repeated Stability of Negative Activation Ratio (RSNAR) represents the proportion of negative responses during the experiment.

$$
\text { RSNAR }=\frac{N_{\text {negative }}}{N}
$$

Where $N_{\text {positive }}$ and $N_{\text {negative }}$ is the time of trials with the positive or negative responses, $N$ is the total time of trials. Reliability Coefficient of Effect in Subjects (AUC' $\boldsymbol{\alpha}$ ) was defined as

$$
\mathrm{AUC}^{\prime} \alpha=\max \left(\max \left(\operatorname{RSPAR}_{c h}, \operatorname{RSNAR}_{c h}\right)\right)
$$

It should be over 0.7 to describe a high-reliability effect for one subject. Generally, $\mathrm{AUC}^{\prime} \alpha>0.7$ means acceptable, $\mathrm{AUC}^{\prime} \alpha>0.8$ means suitable, $\mathrm{AUC}^{\prime} \alpha>0.9$ means excellent.

\section{Experiment1: Simulation-based HRF signal for Response Indicators}

\subsection{Signal Simulation}

Two Gamma functions simulated HRF in SPM12 toolbox/MATLAB R2020a. It was influenced by the delay of response and cut-off timepoint mainly. 


\subsection{Experiment1a: Estimate the Influence of HRF Delay of Response}

Here, we created the simulation-based HRF signal with a different response delay (3 8s) to estimate the influence of delay of response. Again, the default sample rate was set as $1 \mathrm{~Hz}(\mathrm{TR}=1 \mathrm{~s})$.

\subsubsection{Results}

The delay of response can influence all TAD, PAD, PAR, NAR, and NAD, but the influence degree of very small. The result was shown in Fig 2(b).

(a)

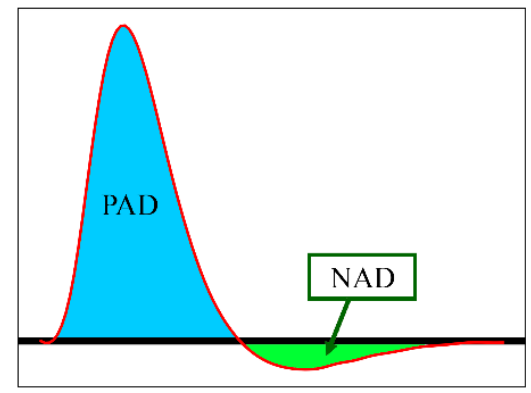

(c)

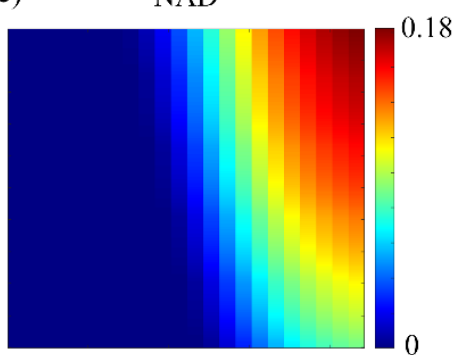

(b)

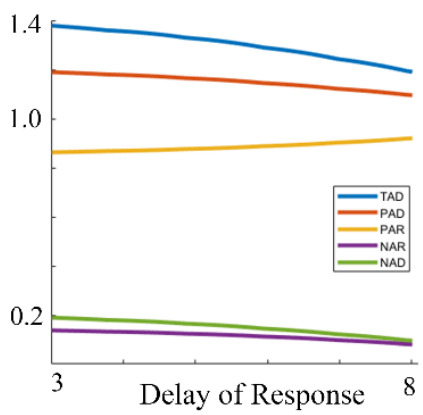

$\mathrm{PAD}$
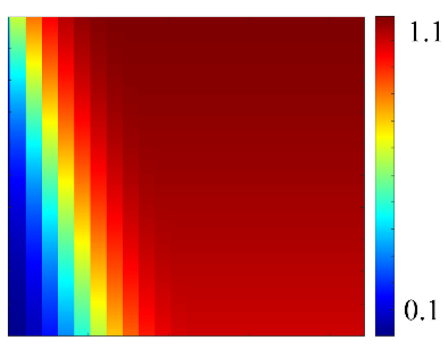

PAR

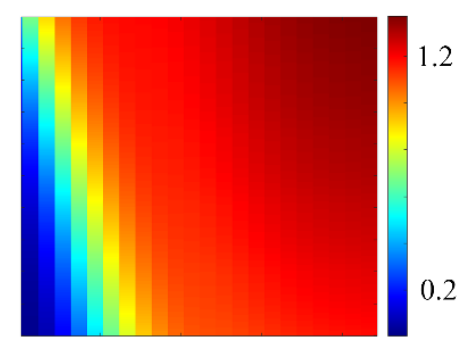

(d)
NAR

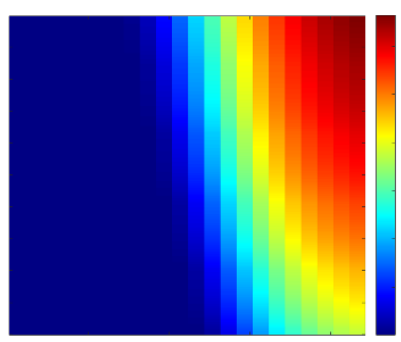

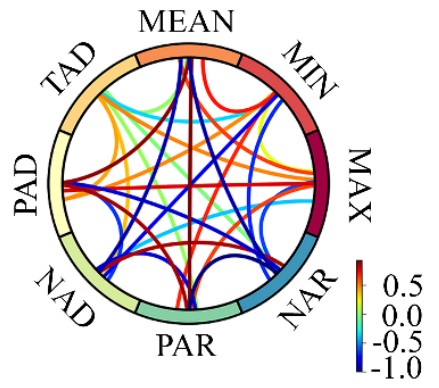

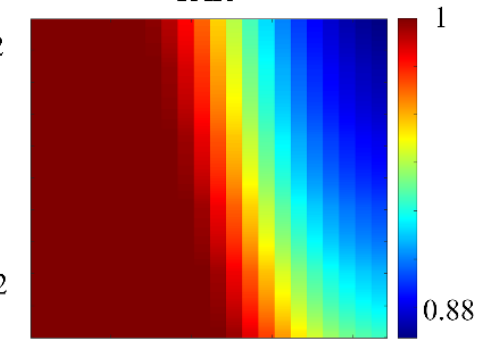

$25 \mathrm{~s}$

Cut-Off Timepoint

Fig 2 (a). The PAD and NAD in HRF. (b). The changes of AUCIS with the different delays of response. (c). The changes of AUCIS with the interaction of different delay of response and cut-off timepoint. (d). The relationship between AUCIS and general indicators.

\subsection{Experiment1b: Estimate the Influence of Cut-Off Timepoint}


Cut-off timepoint can influence the indicators based on the delay of response. Consequently, we created the simulation-based HRF signal with different cut-off timepoints (signal stop at 3 25s early) and delay of response(3 8s) to estimate the influence of delay of Cut-off timepoint.

\subsubsection{Results}

The interaction results were shown in Fig 2(c).

\section{Experiment2: Estimate and Valid the AUCIS in Real Signal}

\subsection{Database}

The fNIRS database was published by mne, recorded at Macquarie University, containing one subject's fNIRS data with a tapping-finger task. It contained 20 channels with 8 sources and 8 detectors, shown in Fig 1(b), to detect the motor cortex's oxyhemoglobin hemodynamics (HbO) and deoxy-hemoglobin hemodynamics (HbR) concentration. The sample rate was $7.8 \mathrm{~Hz}$. During the tasks, there were three conditions, 30 trials tapping the left thumb to fingers, tapping 30 trials tapping the right thumb to fingers, and 30 trials where nothing happens as controls, respectively. Before the task start, a 5-seconds relaxation was asked during a trial. After that, tapping lasted 5 seconds.

\subsection{Preprocessing}

fNIRS data were analyzed by MNE 0.23(Gramfort et al., 2014)/Python 3.9.0. After acquiring raw data as light intensity data, it was transposed to optical density data. Then, the scalp coupling index (sci) was used to evaluate the channel quality of the coupling between the optodes and the scalp(Pollonini et al., 2014). If the sci of the channel were less than 0.5 , the channel would be rejected and interpolated, after which, optical density data was converted to hemoglobin concentration data by beer lambert law, where the partial pathlength factor is 0.1 as $\mathrm{HbO}$ and $\mathrm{HbR}$. A band-pass filter was set after that, and the pass threshold was 0.05 to 0.7 to remove slow drifts and to avoid the unwanted heartbeat wave. Then, the hemoglobin concentration data was split by mark timepoint from -5 second to 15 second. Each fragment subtracted the mean of baseline ( -5 second to 0 second) and then averaged 0 second to 15 second within each condition.

\subsection{Analysis}

\subsubsection{General Indicators Analysis}

The maximum, minimum, and averaged $\mathrm{HbO}$ and $\mathrm{HbR}$ data were calculated as the general indicators for each trial. After that, the general indicators among 30 trials were averaged within every condition as the condition indicators of this subject.

\subsubsection{AUCIS Analysis for Response Indicators}

Firstly, in each trial, PAD was calculated by integrating the data after using the Relu algorithm from 0s to $15 \mathrm{~s}$. Then, NAD was calculated by integrating the opposite data after using the Relu algorithm from 0s to $15 \mathrm{~s}$. Next, TAD was calculated by integrating the absolute maximum, minimum, and averaged $\mathrm{HbO}$ and $\mathrm{HbR}$ data from $0 \mathrm{~s}$ to $15 \mathrm{~s}$. Next, PAR was calculated by dividing PAD from TAD. Then, PAR was calculated by dividing NAD from TAD. Finally, all the response indicators were averaged as the condition indicators of this subject.

\subsubsection{AUCIS Analysis for Reliability Coefficient}

After AUCIS Analysis for Response Indicators, we set the threshold as the range from 0.5 to 0.7 per 0.01 to define the polar of the trials. If the PAR was over the threshold, this trial would be a positive response. If the NAR was over the threshold, this trial would be a negative response. First, the numbers of positive and negative responses were counted, respectively. Then, RSPAR was calculated by dividing the number of positive responses from the total number (30), and RSNAR was calculated by dividing the number of negative responses from the total number (30). Finally, we got paired RSPAR and RSNAR of 3 conditions, respectively. The reliability coefficient (AUC's $\alpha$ ) was defined as the max of them.

\subsubsection{Comparing between General Indicators and AUCIS}

The indicators were calculated first to compare these general and AUCIS indicators, then averaging among conditions and channels. Then, Pearson's correlation coefficient was used to estimate the averaged relationship among the trials. Filter parameters were factors that influent the signal curve. To see the stability of indicators, the band-pass filter from 0.01 to $0.1 \sim 0.7$ was set while calculating the AUCIS and maximum, minimum, and averaged $\mathrm{HbO}$ and $\mathrm{HbR}$ data. 


\subsection{Results}

\subsubsection{General Indicators Results}

The maximum, minimum, and averaged $\mathrm{HbO}$ and $\mathrm{HbR}$ were shown in Fig. In two tapping conditions, the maximum and averaged data showed the markable responses in $\mathrm{HbO}$; the minimum was not clear. However, of averaged $\mathrm{HbO}$, it was clear to identify the difference between the two tagging conditions. The results were shown in Fig 3(a).

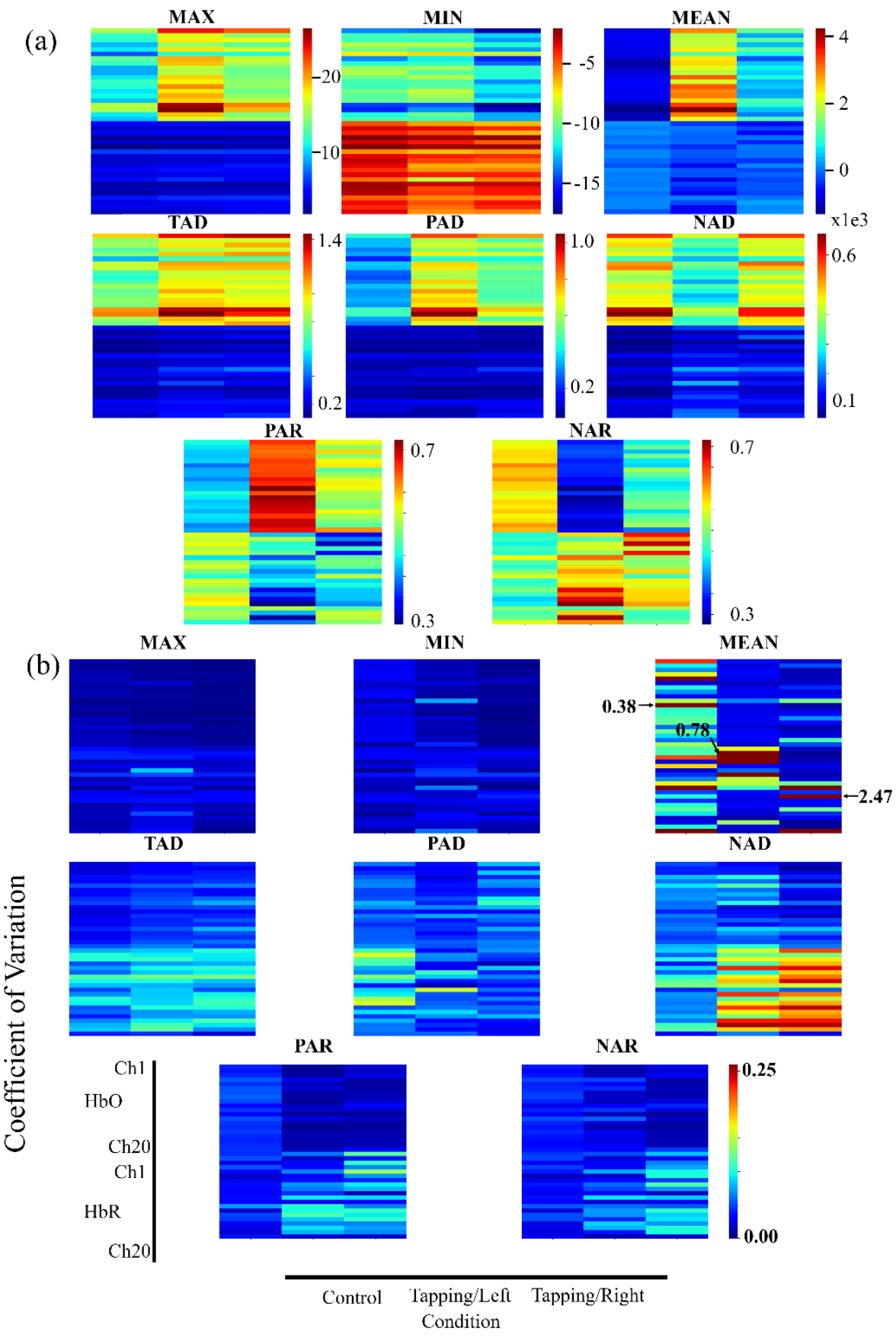

Fig 3 (a). The general indicators and AUCIS value of $\mathrm{HbO}$ and HbR in 20 channels. (b). The coefficient of variation of the general indicators and AUCIS between different band-pass filters. The rows are sorted in order of the first 20 
channels $\mathrm{HbO}$ and then the 20 channels HbR. Columns are sorted by control condition, tapping left finger task, and tapping right finger task.

\subsubsection{AUCIS Results for Response Indicators}

TAD, PAD, NAD, PAR, and NAR were presented in Fig 3(a). As for the control condition, the baseline responses can be anticipated in TAD, PAD, and NAD, which directly describe the activity areas, especially NAD showed a more considerable negative activity. However, PAR and NAR showed the control condition was similar as 0.5 for the most channel. Moreover, the active channels 17 and 18, anticipated by other indicators in the control condition, cannot be identified by PAR and NAR. However, they showed stability from the tagging condition to the control condition in $\mathrm{HbO}$.

\subsubsection{AUCIS Results for Reliability Coefficient}

The AUC's $\alpha$ was shown in Fig 4. It can be anticipated that the left tapping condition had high reliability than others. Moreover, with a lower threshold, the AUC's $\alpha$ tended to be larger.

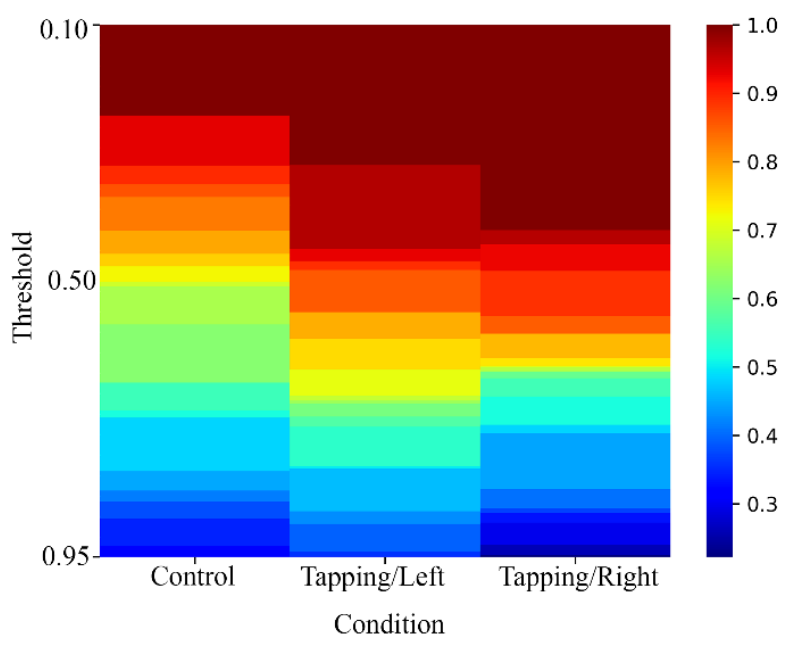

Fig 4 The changing of AUC' $\alpha$ with the different threshold in three conditions, control condition, tapping left finger task and tapping right finger task, respectively.

\subsubsection{Comparing between General and AUCIS}

The robust negative connectivity can be found between NAD, and both averaged and minimum data, respectively. The robust positive connectivity can be found between PAD, PAR, and averaged data, respectively, shown in Fig 2(d). The coefficient of variation results of different filters for AUCIS and maximum, minimum, and averaged $\mathrm{HbO}$ and $\mathrm{HbR}$ data was shown in Fig 3(b). Averaged $\mathrm{HbO}$ and $\mathrm{HbR}$ data was instability across different filters. 


\section{Discussion}

This study established an indicator system based on AUC, named AUCIS, focusing on estimating the reliability coefficient of conditions and explaining the responses occurring in $\mathrm{HbO}$ and $\mathrm{HbR}$ of fNIRS channels. Thus, it first defines the positive response and negative response and, thus, is referred to as a comprehensive physiological mechanism. We also compared it with general indicators, and it showed greater sensitivity and robustness.

In Exp 1a, the influence on the AUCIS was assessed with different delays in response. However, it did not show a marked change during the changing delays in response. During Exp 1b, we considered that most studies cannot wait for the whole HRF to finish, i.e., approximately 30 seconds, and are mostly near $15 \mathrm{~s}$ or less as the time limitation. The dynamic matrix showed that all these indicators could be influenced by the delay in cut-off timepoints but interacted with the delay of the response. Consequently, the time length of the trials using AUCIS should be considered.

In Exp 2, both fMRI(Ersland et al., 1996; Khorrami et al., 2011; Zang et al., 2004) and EEG(Salmelin et al., 1995; Urbano et al., 1998; Ziemann \& Hallett, 2001) studies have shown that finger tapping improved the motor cortex response, and this was also detected by both the general indicators and AUCIS in this study. The maximum and average amplitudes showed that the amplitudes while tapping the left and right fingers were higher than those in the control condition, especially the left finger; however, the minimum amplitude was not sensitive in this study because it was a positive response, as evaluated by PAR and NAR. The averaged amplitude with the filters was sensitive. These measures showed high coefficients of variation, such as 2.47 in HbR of channel 12 and 0.38 in $\mathrm{HbO}$ of channel 11.

Continuing to compare these three conditions, the response robustness could be estimated by AUC' $\alpha$, and the reliability of the control condition was lower than that of the other conditions. Mostly, the experimental condition presented a greater than $0.8 \mathrm{AUC}^{\prime} \alpha$, showing high robustness within the finger-tapping conditions. The AUCIS was stable with different filters and showed robust effects.

Taken together, the AUCIS response indicators were more robust than the general indicators, and distinguishing between different responses became possible. The reliability coefficient of the condition (AUC' $\alpha$ ) within the AUCIS can provide the robustness for each condition, thus allowing a determination of whether the subject was effectively activated by the task. The AUCIS also presented additional explanations for performance in the cognitive task. Combining $\mathrm{HbO}$ and $\mathrm{HbR}$, both the positive response and negative response as part of a comprehensive physiological mechanism can be detected. A likely explanation(N. Tam et al., 2017; N. D. Tam \& Zouridakis, 2014) for this involves the fact that a positive response of $\mathrm{HbO}$ is considered an improvement in oxygen delivery; in contrast, a negative response is considered an impediment. A positive response of HbR is considered an improvement in oxygen extraction; in contrast, a negative response is considered an impeding response. This is also one orientation in which fNIRS application could be superior to fMRI, as it detects more physiological responses in the time field.

Moreover, there are some guidelines for the entire methodology. Fig 1(c) showed the processing.

(1) All AUCIS indicators should be calculated after preprocessing. Removing motion artifacts with a motion correction method and setting a rejection threshold to reject abnormal and extreme trial signals are necessary; here, we recommend setting a rejection threshold or temporal derivative distribution repair (TDDR)(Fishburn et al., 2019). The rejection threshold is dependent on the task design.

(2) The AUC's $\alpha$ threshold is mostly recommended to be set as 0.5 .

(3) Before statistical analysis, the AUC' $\alpha$ should be considered: if all conditions show a low AUC' $\alpha$ value, this subject should be rejected. If no subject has a high AUC' $\alpha$, this experiment may fail because it does not show a robust effect.

(4) Mostly, $\mathrm{HbO}$ and $\mathrm{HbR}$ should be considered equally and reported. Depending on the AUCIS, both $\mathrm{HbO}$ and $\mathrm{HbR}$ can provide proof of physiological mechanisms. Although the physiological mechanism may not be clearly explained at this time, it may be analyzed by review and meta-analysis in the future. 


\section{Reference}

Asgher, U., Ahmad, R., Naseer, N., Ayaz, Y., Khan, M. J., \& Amjad, M. K. (2019). Assessment and Classification of Mental Workload in the Prefrontal Cortex (PFC) Using Fixed-Value Modified Beer-Lambert Law. IEEE Access, 7, 143250-143262. https://doi.org/10.1109/ACCESS.2019.2944965

Baron, J.-C., \& Jones, T. (2012). Oxygen metabolism, oxygen extraction and positron emission tomography: Historical perspective and impact on basic and clinical neuroscience. NeuroImage, 61(2), $492-504$. https://doi.org/10.1016/j.neuroimage.2011.12.036

Brazy, J. E., Lewis, D. V., Mitnick, M. H., \& Jöbsis vander Vliet, F. F. (1985). Noninvasive monitoring of cerebral oxygenation in preterm infants: Preliminary observations. Pediatrics, 75(2), 217-225.

Calderon-Arnulphi, M., Alaraj, A., \& Slavin, K. V. (2009). Near infrared technology in neuroscience: Past, present and future. Neurological Research, 31(6), 605-614. https://doi.org/10.1179/174313209X383286

Cope, M., Delpy, D. T., Reynolds, E. O. R., Wray, S., Wyatt, J., \& van der Zee, P. (1988). Methods of Quantitating Cerebral Near Infrared Spectroscopy Data. In M. Mochizuki, C. R. Honig, T. Koyama, T. K. Goldstick, \& D. F. Bruley (Eds.), Oxygen Transport to Tissue $X$ (Vol. 222, pp. 183-189). Springer US. https://doi.org/10.1007/978-1-4615-9510-6_21

Curtin, A., Ayaz, H., Tang, Y., Sun, J., Wang, J., \& Tong, S. (2019). Enhancing neural efficiency of cognitive processing speed via training and neurostimulation: An fNIRS and TMS study. NeuroImage, 198, 73-82. https://doi.org/10.1016/j.neuroimage.2019.05.020

De Blasi, R. A., Cope, M., Elwell, C., Safoue, F., \& Ferrari, M. (1993). Noninvasive measurement of human forearm oxygen consumption by near infrared spectroscopy. European Journal of Applied Physiology and Occupational Physiology, 67(1), 20-25. https://doi.org/10.1007/BF00377698

Ersland, L., Rosén, G., Lundervold, A., Smievoll, A. I., Tillung, T., Sundberg, H., \& ugdahl, K. (1996). Phantom limb imaginary fingertapping causes primary motor cortex activation: An fMRI study. NeuroReport, 8(1), 207210. https://doi.org/10.1097/00001756-199612200-00042 
Fan, A. P., An, H., Moradi, F., Rosenberg, J., Ishii, Y., Nariai, T., Okazawa, H., \& Zaharchuk, G. (2020). Quantification of brain oxygen extraction and metabolism with [15O]-gas PET: A technical review in the era of PET/MRI. NeuroImage, 220, 117136. https://doi.org/10.1016/j.neuroimage.2020.117136

Fishburn, F. A., Ludlum, R. S., Vaidya, C. J., \& Medvedev, A. V. (2019). Temporal Derivative Distribution Repair (TDDR): A motion correction method for fNIRS. NeuroImage, 184, 171-179. https://doi.org/10.1016/j.neuroimage.2018.09.025

Gramfort, A., Luessi, M., Larson, E., Engemann, D. A., Strohmeier, D., Brodbeck, C., Parkkonen, L., \& Hämäläinen, M. S. (2014). MNE software for processing MEG and EEG data. NeuroImage, 86, 446-460. https://doi.org/10.1016/j.neuroimage.2013.10.027

Grossmann, T., Missana, M., \& Krol, K. M. (2018). The neurodevelopmental precursors of altruistic behavior in infancy. PLOS Biology, 16(9), e2005281. https://doi.org/10.1371/journal.pbio.2005281

Hoshi, Y. (2011). Towards the next generation of near-infrared spectroscopy. Philosophical Transactions of the Royal Society A: Mathematical, Physical and Engineering Sciences, 369(1955), 4425-4439. https://doi.org/10.1098/rsta.2011.0262

Kaufman, D. P., Kandle, P. F., Murray, I., \& Dhamoon, A. S. (2021). Physiology, Oxyhemoglobin Dissociation Curve. In StatPearls. StatPearls Publishing. http://www.ncbi.nlm.nih.gov/books/NBK499818/

Khorrami, M. S., Faro, S. H., Seshadri, A., Moonat, S., Lidicker, J., Hershey, B. L., \& Mohamed, F. B. (2011). Functional MRI of Sensory Motor Cortex: Comparison Between Finger-to-Thumb and Hand Squeeze Tasks. Journal of Neuroimaging, 21(3), 236-240. https://doi.org/10.1111/j.1552-6569.2010.00492.x

Logothetis, N. K., Pauls, J., Augath, M., Trinath, T., \& Oeltermann, A. (2001). Neurophysiological investigation of the basis of the fMRI signal. Nature, 412(6843), 150-157. https://doi.org/10.1038/35084005

Mayseless, N., Hawthorne, G., \& Reiss, A. L. (2019). Real-life creative problem solving in teams: FNIRS based hyperscanning study. NeuroImage, 203, 116161. https://doi.org/10.1016/j.neuroimage.2019.116161

Montcel, B., Chabrier, R., \& Poulet, P. (2006). Time-resolved absorption and hemoglobin concentration difference maps: A method to retrieve depth-related information on cerebral hemodynamics. Optics Express, 14(25), 12271. https://doi.org/10.1364/OE.14.012271 
Ochi, G., Yamada, Y., Hyodo, K., Suwabe, K., Fukuie, T., Byun, K., Dan, I., \& Soya, H. (2018). Neural basis for reduced executive performance with hypoxic exercise. NeuroImage, 171, 75-83. https://doi.org/10.1016/j.neuroimage.2017.12.091

Okazawa, H., \& Kudo, T. (2009). Clinical impact of hemodynamic parameter measurement for cerebrovascular disease using positron emission tomography and (15)O-labeled tracers. Annals of Nuclear Medicine, 23(3), 217-227. https://doi.org/10.1007/s12149-009-0235-7

Pellicer, A., \& Bravo, M. del C. (2011). Near-infrared spectroscopy: A methodology-focused review. Seminars in Fetal and Neonatal Medicine, 16(1), 42-49. https://doi.org/10.1016/j.siny.2010.05.003

Poldrack, R. A., Mumford, J. A., \& Nichols, T. E. (2011). Handbook of Functional MRI Data Analysis. Handbook of Functional MRI Data Analysis.

Pollonini, L., Olds, C., Abaya, H., Bortfeld, H., Beauchamp, M. S., \& Oghalai, J. S. (2014). Auditory cortex activation to natural speech and simulated cochlear implant speech measured with functional near-infrared spectroscopy. Hearing Research, 309, 84-93. https://doi.org/10.1016/j.heares.2013.11.007

Rosa, E. D., Brigadoi, S., Cutini, S., Tarantino, V., Dell’Acqua, R., Mapelli, D., Braver, T. S., \& Vallesi, A. (2019). Reward motivation and neurostimulation interact to improve working memory performance in healthy older adults: A simultaneous tDCS-fNIRS study. NeuroImage, 202, 116062. https://doi.org/10.1016/j.neuroimage.2019.116062

Rovetti, J., Goy, H., Pichora-Fuller, M. K., \& Russo, F. A. (2019). Functional Near-Infrared Spectroscopy as a Measure of Listening Effort in Older Adults Who Use Hearing Aids. Trends in Hearing, 23, 233121651988672. https://doi.org/10.1177/2331216519886722

Sagiv, S. K., Bruno, J. L., Baker, J. M., Palzes, V., Kogut, K., Rauch, S., Gunier, R., Mora, A. M., Reiss, A. L., \& Eskenazi, B. (2019). Prenatal exposure to organophosphate pesticides and functional neuroimaging in adolescents living in proximity to pesticide application. Proceedings of the National Academy of Sciences, 116(37), 18347-18356. https://doi.org/10.1073/pnas.1903940116 
Salmelin, R., Forss, N., Knuutila, J., \& Hari, R. (1995). Bilateral activation of the human somatomotor cortex by distal hand movements. Electroencephalography and Clinical Neurophysiology, 95(6), 444-452. https://doi.org/10.1016/0013-4694(95)00193-X

Tam, N. D., \& Zouridakis, G. (2013). Decoding of movement direction using optical imaging of motor cortex. BMC Neuroscience, 14(S1), P380, 1471-2202-14-S1-P380. https://doi.org/10.1186/1471-2202-14-S1-P380

Tam, N. D., \& Zouridakis, G. (2014). Temporal decoupling of oxy- and deoxy-hemoglobin hemodynamic responses detected by functional near-infrared spectroscopy (fNIRS). Journal of Biomedical Engineering and Medical Imaging, 1(2), 18-28. https://doi.org/10.14738/jbemi.12.146

Tam, N., Pollonini, L., \& Zouridakis, G. (2017). Computational Method for Representing the Simultaneous Hemodynamic Relationship between Oxy-Hemoglobin Delivery and Deoxy-Hemoglobin Extraction in Neural Tissues. American Journal of Biomedical Science and Engineering, 3(6), 54-63.

Tam, N., \& Zouridakis, G. (2013). Optical imaging of motor cortical hemodynamic response to directional arm movements using near-infrared spectroscopy. International Journal of Biological Engineering, 3, 11-17. https://doi.org/10.5923/j.ijbe.20130302.01

Toronov, V., Webb, A., Choi, J. H., Wolf, M., Michalos, A., Gratton, E., \& Hueber, D. (2001). Investigation of human brain hemodynamics by simultaneous near-infrared spectroscopy and functional magnetic resonance imaging. Medical Physics, 28(4), 521-527. https://doi.org/10.1118/1.1354627

Tse, C.-Y., Yip, L.-Y., Lui, T. K.-Y., Xiao, X.-Z., Wang, Y., Chu, W. C. W., Parks, N. A., Chan, S. S.-M., \& Neggers, S. F. W. (2018). Establishing the functional connectivity of the frontotemporal network in pre-attentive change detection with Transcranial Magnetic Stimulation and event-related optical signal. NeuroImage, 179, 403-413. https://doi.org/10.1016/j.neuroimage.2018.06.053

Urbano, A., Babiloni, C., Onorati, P., Carducci, F., Ambrosini, A., Fattorini, L., \& Babiloni, F. (1998). Responses of human primary sensorimotor and supplementary motor areas to internally triggered unilateral and simultaneous bilateral one-digit movements. A high-resolution EEG study. European Journal of Neuroscience, 10(2), 765-770. https://doi.org/10.1046/j.1460-9568.1998.00072.x 
Wang, X., He, Y., Lu, K., Deng, C., Qiao, X., \& Hao, N. (2019). How does the embodied metaphor affect creative thinking? NeuroImage, 202, 116114. https://doi.org/10.1016/j.neuroimage.2019.116114

Yablonskiy, D. A., Sukstanskii, A. L., \& He, X. (2013). Blood oxygenation level-dependent (BOLD)-based techniques for the quantification of brain hemodynamic and metabolic properties - theoretical models and experimental approaches: CEREBRAL HEMODYNAMICS AND METABOLISM. NMR in Biomedicine, 26(8), 963986. https://doi.org/10.1002/nbm.2839

Ye, J., Tak, S., Jang, K., Jung, J., \& Jang, J. (2009). NIRS-SPM: Statistical parametric mapping for near-infrared spectroscopy. NeuroImage, 44(2), 428-447. https://doi.org/10.1016/j.neuroimage.2008.08.036

Yuan, Y., Li, G., Ren, H., \& Chen, W. (2020). Caffeine Effect on Cognitive Function during a Stroop Task: FNIRS Study. Neural Plasticity, 2020, 1-8. https://doi.org/10.1155/2020/8833134

Zang, Y., Jiang, T., Lu, Y., He, Y., \& Tian, L. (2004). Regional homogeneity approach to fMRI data analysis. NeuroImage, 22(1), 394-400. https://doi.org/10.1016/j.neuroimage.2003.12.030

Ziemann, U., \& Hallett, M. (2001). Hemispheric asymmetry of ipsilateral motor cortex activation during unimanual motor tasks: Further evidence for motor dominance. Clinical Neurophysiology, 112(1), $107-113$. https://doi.org/10.1016/S1388-2457(00)00502-2 